\title{
Avaliação prognóstica dos aneurismas intracranianos por estudo do grau clínico, tomografia computadorizada e angiografia cerebral
}

\author{
Pasquale Gallo*, Antonio de Pádua Bonatelli** \\ Serviço de Neurocirurgia do Hospital Cristo Redentor, Porto Alegre, RS \\ Escola Paulista de Medicina, UNIFESP, São Paulo, SP
}

\section{RESUMO}

Embora sejam inúmeras as publicações sobre o prognóstico dos aneurismas intracranianos utilizando uma metodologia cada vez mais sofisticada, este trabalho tende a demonstrar que os parâmetros mais elementares são os mais confiáveis para o prognóstico.

Com a finalidade de compreender e quantificar esse prognóstico, foram estudados prospectivamente 100 pacientes com hemorragia subaracnóidea provocada por aneurismas intracranianos, confirmado por diversos métodos diagnósticos no Serviço de Neurocirurgia do Hospital Cristo Redentor, em Porto Alegre, entre outubro de 1992 e outubro de 1995. Todos os pacientes foram classificados pelo grau clínico à internação, pela presença ou pela ausência de sangue na tomografia computadorizada e pela presença ou pela ausência de vasoespasmo angiográfico. Os exames foram realizados tão precocemente quanto possível, de acordo com o estado clínico do paciente. A cirurgia foi realizada assim que as condições clínicas permitiram. Os pacientes foram seguidos pelo período de seis meses e os resultados correlacionados aos diversos fatores que foram analisados.

Foi possível constatar que a correta observação do grau clínico, da tomografia computadorizada e da angiografia cerebral, tanto isoladamente quanto em conjunto, nos forneceu uma quantidade expressiva de informações, capaz de nos indicar o prognóstico do resultado pós-operatório de forma bastante confiável.

\section{PALAVRAS-CHAVE}

Aneurisma. Angiografia. Tomografia computadorizada.

\section{ABSTRACT}

The value of the clinical grade and the findings in computed tomography and cerebral angiography to predict prognosis for intracranial aneurysms

In an era of increasing limitations of medical resources, the ability to predict outcome is becoming increasingly important because when the outcome can be accurately predicted, treatment is more likely to be geared toward potentially avoidable, deleterious factors. The best factors to predict outcome are the most commom.

A prospective analysis of 100 patients with aneurysms treated at the Neurosurgical Service, Hospital Cristo Redentor, Porto Alegre, during the period from October 1992 to October 1995, was carried out.

All patients were classified and scored according to Hunt \& Hess scale, the amount and localization of blood shown in the computed tomography, and presence or absence of angiographic vasospasm. The timing of surgical intervention was, in general, determined by the patient's neurological function, and the follow-up was done for six months. Outcome was reviewed by multifactors analysis.

Excellent results were verified in $71 \%$, good results in $19 \%$ and poor results in $9 \%$. The mortality was $1 \%$. The preoperative overall score obtained by the patients based on the clinical grade, computerized tomography and cerebral angiography findings was directly related to the outcome.

\section{KEYWORDS}

Aneurysms. Angiography. Computed tomography.

\footnotetext{
* Mestre em Neurocirurgia e Doutor em Medicina pela Universidade Federal de São Paulo.

** Doutor em Medicina e Professor-adjunto da Universidade Federal de São Paulo.
} 


\section{Introdução}

O tratamento cirúrgico dos aneurismas intracranianos sofreu, a partir da década de 1960, transformações importantes que se refletiram favoravelmente nos resultados obtidos a partir desse momento. Essas modificações foram observadas nas modernas técnicas microneurocirúrgicas, como o uso do microscópio operatório, o instrumental microcirúrgico e a coagulação bipolar. Por outro lado, o melhor conhecimento da microanatomia vascular e o emprego de agentes anestésicos mais confiáveis deram ao neurocirurgião maior segurança durante o ato operatório.

Estudos cooperativos e estatísticos, nos Estados Unidos, mostram que, anualmente, 28 mil novos casos de aneurismas são computados e que somente 9 mil desses pacientes retornam às suas atividades anteriores, e 2 mil morrem por complicações cirúrgicas ${ }^{49}$.

Menos de $40 \%$ dos pacientes que têm hemorragia subaracnóidea (HSA) por ruptura aneurismática sobrevivem sem danos funcionais de grande repercussão ${ }^{50}$. Os fatores mais importantes que determinam a mortalidade e a morbidade são a intensidade da hemorragia inicial, a incidência de ressangramento e a ocorrência de vasoespasmo $0^{50,52,53,54,64,65,70,75}$.

A controvérsia entre a preferência pela cirurgia precoce, preconizada com o objetivo de evitar o ressangramento e permitir tratamento agressivo do vasoespasmo $^{7,8,9,11,12,28,34,35,44,58,63,78,98}$ ou pela cirurgia tardia, com seus bons resultados ${ }^{4,18,59,65,106,109}$, permanece. Entretanto, não foi confirmada a melhora nos resultados em pacientes sonolentos ou comatosos operados precocemente ${ }^{19,53,54}$.

Portanto, apesar dos recentes desenvolvimentos nas técnicas microcirúrgicas para os aneurismas cerebrais e o tratamento do vasoespasmo, o prognóstico do paciente que sofre HSA permanece insatisfatório ${ }^{53,54,76,82,105}$ e os resultados cirúrgicos dependem, principalmente, das condições neurológicas e do nível de consciência do paciente ${ }^{2,65,103}$.

No entanto, mesmo aqueles pacientes que são operados em boas condições e mais tardiamente, após a hemorragia, podem apresentar resultados insatisfatórios, havendo necessidade de mais estudos sobre os fatores que podem influenciar no resultado da cirurgia dos aneurismas intracranianos.

Com o objetivo de determinar outros fatores prognósticos, avaliamos quantitativamente os pacientes que sofreram HSA por ruptura de aneurisma intracraniano e que apresentavam boas condições neurológicas e, principalmente, nível de consciência considerado ideal para a realização do procedimento cirúrgico, independentemente do número de dias que transcorreram desde a HSA.
A avaliação desses pacientes foi realizada levandose em consideração: o grau clínico (GC), as alterações encontradas na tomografia computadorizada cerebral (TC) e na angiografia cerebral (AC).

\section{Casuística e métodos}

O material clínico utilizado neste trabalho é proveniente do Serviço de Neurocirurgia do Hospital Cristo Redentor, da cidade de Porto Alegre, no Rio Grande do Sul. No período compreendido entre outubro de 1992 e outubro de 1995 foram protocolados e estudados prospectivamente 100 pacientes com aneurismas intracranianos, que são objeto e análise deste estudo. Foram excluídos deste estudo pacientes que apresentavam possíveis alterações anatômicas, ou fatores clínicos que pudessem comprometer a uniformidade do trabalho, como:

a) pacientes em grau $\mathrm{V}^{40}$;

b) pacientes que haviam sofrido craniotomias prévias por outras lesões intracranianas;

c) pacientes com comprometimento clínico de outros órgãos ou sistemas que pudessem influenciar o resultado cirúrgico.

Todos os 100 pacientes foram submetidos ao protocolo especialmente realizado para este trabalho, recebendo conduta neurológica e tratamento uniforme, destacando-se que: todos foram operados pelo mesmo cirurgião (P.G.); a abordagem cirúrgica mais utilizada foi a pterional ou frontotemporoesfenoidal ${ }^{106}$, adotada em todos os pacientes com aneurismas da circulação anterior, com exceção dos seis casos de aneurismas da artéria pericalosa que foram abordados por via interhemisférica ${ }^{106}$; em um paciente com aneurisma da artéria cerebelosa póstero-inferior foi utilizada a via suboccipital ${ }^{106}$; todos os pacientes foram tratados e operados conforme a seqüência de internação; finalmente, todos os pacientes foram avaliados e receberam uma pontuação de acordo com os critérios abaixo, no momento da internação, e os exames tomográficos e angiográficos foram obtidos o mais precocemente possível, tanto quanto as condições clínicas do paciente o permitiram. 1968):

Pontuação segundo o grau clínico (Hunt \& $\mathrm{Hess}^{40}$,

$$
\begin{aligned}
& \mathrm{I}=4 \text { pontos } \\
& \mathrm{II}=3 \text { pontos } \\
& \mathrm{III}=2 \text { pontos } \\
& \mathrm{IV}=1 \text { ponto } \\
& \mathrm{V}=0 \text { ponto }
\end{aligned}
$$


Pontuação segundo a tomografia computadorizada:

Presença de hematoma $=0$ ponto

Presença de sangue intraventricular $=1$ ponto

Presença de sangue nas cisternas $=2$ pontos

Ausência de sangue $=3$ pontos

Pontuação segundo a angiografia cerebral:

Com vasoespasmo $=0$ ponto

Sem vasoespasmo $=1$ ponto

\section{Resultados}

Os resultados do tratamento estão apresentados nas tabelas a seguir, tendo sido protocolados um total de 100 pacientes $(n=100)$ que apresentavam 103 aneurismas intracranianos, salientando-se que os pacientes foram observados e acompanhados durante período de até 6 meses após a cirurgia.

O resultado pós-operatório foi avaliado classificando-se os pacientes em três grupos (excelente, bom e mau), de acordo com o seu estado clínico final, utilizando-se a classificação de Jomin e cols. ${ }^{48}$

\begin{tabular}{|c|c|c|}
\hline \multicolumn{3}{|c|}{$\begin{array}{c}\text { Tabela } 1 \\
\text { Distribuição dos doentes segundo o grau clínico }\end{array}$} \\
\hline Grau & $\mathbf{N}$ & $\%$ \\
\hline I & 46 & 46 \\
\hline II & 21 & 21 \\
\hline III & 29 & 29 \\
\hline IV & 4 & 4 \\
\hline Total & 100 & 100 \\
\hline
\end{tabular}

Tabela 2

Sangue detectado na TC

$\mathrm{N}^{*} \quad \%$

\begin{tabular}{lll}
\hline Hematoma & 12 & 12 \\
Intraventricular & 21 & 21 \\
Cisternas & 60 & 60 \\
Ausência & 34 & 34 \\
\hline
\end{tabular}

* Em 21 a TC apresentava associação de sangue nas cisternas e intraventricular e/ou hematomas

\begin{tabular}{lcc}
\hline \multicolumn{3}{c}{ Tabela } \\
Localização & dos & aneurismas \\
\hline Sítio & N & $\%$ \\
\hline Comunicante posterior* & 31 & 30,5 \\
Carótida interna & 12 & 11,6 \\
Comunicante anterior & 29 & 28,1 \\
Cerebral média & 22 & 21,3 \\
Pericalosa & 6 & 5,8 \\
Coroidéia anterior* & 1 & 0,9 \\
Oftálmica & 1 & 0,9 \\
Cerebelar póstero-inferior & 1 & 0,9 \\
\hline Total & 103 & 100 \\
* São considerados aneurismas da artéria comunicante posterior e da \\
coroidéia anterior os aneurismas que se situam próximos ou no óstio das \\
respectivas artérias.
\end{tabular}

\begin{tabular}{lcccc}
\hline \multicolumn{5}{c}{ Tabela } \\
& Complicações & e resultados \\
\hline Complicação & Excelente & B o m & Mau & Total \\
\hline Vasoespasmo & - & - & 1 & 1 \\
Hidrocefalia & 1 & 1 & 2 & 4 \\
Meningite & 1 & - & - & 1 \\
Emb.pulm. & 1 & - & - & 1 \\
Der.pleural & 1 & - & - & 1 \\
Convulsões & 1 & - & - & 1 \\
Infec.pele & 2 & - & 1 & 3 \\
Infec.resp. & 1 & - & - & 1 \\
\hline Total & $8(61,5 \%)$ & $1(7,7 \%)$ & $4(30,8 \%)$ & 13 \\
\hline
\end{tabular}

\begin{tabular}{lcccc}
\hline \multicolumn{5}{c}{ Tabela } \\
Vasoespasmo & angiográfico & e resultados \\
\hline & Excelente & B o m & Mau & Total \\
\hline $\mathrm{N}^{\circ}$ pacientes & 12 & 5 & 6 & 23 \\
$\%$ & 52,2 & 21,7 & 26,1 & 100 \\
\hline
\end{tabular}




\begin{tabular}{lcccc}
\hline \multicolumn{5}{c}{ Tabela 7} \\
& Resultados de acordo com & a faixa etária \\
\hline $\begin{array}{l}\text { Idade } \\
\text { (a nos) }\end{array}$ & Excelente & Bo m & Mau & Total \\
\hline 10 a 19 & $1(100 \%)$ & - & - & 1 \\
20 a 29 & $5(62,5 \%)$ & $2(25 \%)$ & $1(12,5 \%)$ & 8 \\
30 a 39 & $15(75 \%)$ & $4(20 \%)$ & $1(5 \%)$ & 20 \\
40 a 49 & $26(78,8 \%)$ & $4(12,2 \%)$ & $3(9 \%)$ & 33 \\
50 a 59 & $16(64 \%)$ & $6(24 \%)$ & $3(12 \%)$ & 25 \\
60 a 69 & $7(70 \%)$ & $2(20 \%)$ & $1(10 \%)$ & 10 \\
70 a 79 & $2(66,6 \%)$ & $1(33,4 \%)$ & - & 3 \\
\hline Total & 72 & 19 & 9 & 100 \\
\hline
\end{tabular}

\begin{tabular}{ccccc}
\hline \multicolumn{5}{c}{ Tabela 8} \\
& Resultados de acordo com o grau clínico \\
\hline Grau & Excelente & B o m & Mau & Total \\
\hline I & $44(95,6 \%)$ & $2(4,4 \%)$ & - & 46 \\
II & $17(80,9 \%)$ & $4(19,1 \%)$ & - & 21 \\
III & $10(34,5 \%)$ & $13(44,8 \%)$ & $6(20,7 \%)$ & 29 \\
IV & $1(25 \%)$ & - & $3(75 \%)$ & 4 \\
\hline Total & 72 & 19 & 9 & 100 \\
\hline
\end{tabular}

\section{Tabela 9}

Resultados de acordo com a localização do aneurisma

\begin{tabular}{lcccc}
\hline Localizaçã o & Excelente & Bo m & Mau & Total \\
\hline Carótida interna & $9(75 \%)$ & $3(25 \%)$ & - & 12 \\
Com. anterior & $23(79,3 \%)$ & $3(10,3 \%)$ & $3(10,4 \%)$ & 29 \\
Cerebral média & $10(45,5 \%)$ & $7(31,8 \%)$ & $5(22,7 \%)$ & 22 \\
Coroidéia ant. & $1(100 \%)$ & - & - & 1 \\
Pericalosa & $5(83,3 \%)$ & $1(16,7 \%)$ & - & 6 \\
Oftálmica & $1(100 \%)$ & - & - & 1 \\
Com. posterior & $24(75,5 \%)$ & $6(19,4 \%)$ & $1(3,1 \%)$ & 31 \\
Cereb. post. inf. & $1(100 \%)$ & - & - & 1 \\
\hline Total & 74 & 20 & 9 & 103 \\
\hline
\end{tabular}

\begin{tabular}{ccccc}
\hline \multicolumn{5}{c}{ Tabela $\mathbf{1 0}$} \\
Resultados de acordo com & a pontuação & pelo grau & clínico \\
\hline Pontuação & Excelente & Bo m & Mau & Total \\
\hline 1 & $1(25 \%)$ & - & $3(75 \%)$ & 4 \\
2 & $10(34,5 \%)$ & $13(44,8 \%)$ & $6(20,7 \%)$ & 29 \\
3 & $16(80 \%)$ & $4(20 \%)$ & - & 20 \\
4 & $45(95,7 \%)$ & $2(4,3 \%)$ & - & 47 \\
\hline Total & 72 & 19 & 9 & 100 \\
\hline
\end{tabular}

Tabela 11

Resultados de acordo com a pontuação da TC

\begin{tabular}{ccccc}
\hline Pontuação & Excelente & Bo m & Mau & Total \\
\hline 0 & $3(25 \%)$ & $8(66,6 \%)$ & $1(8,4 \%)$ & 12 \\
1 & $11(64,7 \%)$ & $2(11,8 \%)$ & $4(23,5 \%)$ & 17 \\
2 & $29(78,3 \%)$ & $5(13,5 \%)$ & $3(8,2 \%)$ & 37 \\
3 & $29(85,3 \%)$ & $4(11,8 \%)$ & $1(2,9 \%)$ & 34 \\
\hline Total & 72 & 19 & 9 & 100 \\
\hline
\end{tabular}

Tabela 12

Resultados de acordo com a pontuação na $A C$

\begin{tabular}{ccccc}
\hline Pontuação & Excelente & Bom & Mau & Total \\
\hline 0 & $13(56,5 \%)$ & $5(21,7 \%)$ & $5(21,8 \%)$ & 23 \\
1 & $59(76,6 \%)$ & $14(18,2 \%)$ & $4(5,2 \%)$ & 77 \\
\hline Total & 72 & 19 & 9 & 100 \\
\hline
\end{tabular}

Tabela 13

Resultados após a soma dos critérios de pontuação

\begin{tabular}{ccccc}
\hline Pontuação & Excelente & Bom & Mau & Total \\
\hline 1 & - & - & - & - \\
2 & $1(33,3 \%)$ & $1(33,3 \%)$ & $1(33,3 \%)$ & 3 \\
3 & - & $7(58,3 \%)$ & $5(41,7 \%)$ & 12 \\
4 & $6(50 \%)$ & $4(33,3 \%)$ & $2(16,7 \%)$ & 12 \\
5 & $6(75 \%)$ & $1(12,5 \%)$ & $1(12,5 \%)$ & 8 \\
6 & $21(91,3 \%)$ & $2(8,7 \%)$ & - & 23 \\
7 & $21(84 \%)$ & $4(16 \%)$ & - & 25 \\
8 & $17(100 \%)$ & - & - & 17 \\
\hline Total & 72 & 19 & 9 & 100 \\
\hline
\end{tabular}

\section{Discussão}

Analisando os 100 pacientes que foram objeto deste estudo, notamos que foram protocolados e operados $31(31 \%)$ homens e $69(69 \%)$ mulheres, o que corresponde, aproximadamente, aos dados encontrados na literatura, referentes aos aneurismas intracranianos $\operatorname{rotos}^{47,53,65,86,100,106}$.

Em relação à idade, o paciente mais jovem no nosso estudo tinha 17 anos e o de maior idade 72 anos, ficando a média em 45 anos. A maior incidência ocorreu na quarta, na quinta e na sexta décadas com 78 pacientes e equivalentes a $78 \%$ dos casos, com o pico máximo na quinta década, representando $33 \%$ do total. Esses dados são coincidentes com diversos autores ${ }^{47,53,65,71,86,106}$. Yoshimoto e cols. ${ }^{109}$, revisando mil aneurismas operados, encontraram o pico máximo de incidência na sexta década, com 32\%. Abaixo dos 20 anos, tivemos, em nossa série, somente 1 caso (1\%), enquanto Yoshimoto e cols. ${ }^{109}$ encontraram $0,5 \%$ e Suriano ${ }^{101}, 3,5 \%$. É interessante notar que em nossa casuística encontram-se 3 pacientes (3\%) pertencentes à oitava década, idade compreendida entre 70 e 79 anos, demonstrando a tendência atual de se operar pacientes mais idosos em boas condições, não sendo mais a idade fator limitante do tratamento cirúrgico e dos resultados ${ }^{5,29,39,42,43,80}$.

É importante estimar o grau clínico de cada paciente com HSA, porque trata-se de correlacionar o estado logo após a HSA e os resultados pós-cirúrgicos. Constatamos na tabela 1 que, ao serem protocolados, os nossos pacientes foram enquadrados pela escala de Hunt \& Hess conforme a seguinte distribuição: $46 \%$ 
em grau I, 21\% em grau II e 29\% em grau III (totalizando $96 \%$ nos graus I, II e III) e somente $4 \%$ em grau IV. Nenhum dos nossos pacientes em grau $\mathrm{V}$ foi levado à cirurgia. Le Roux e cols. ${ }^{62}$, em estudo recente, denominaram 224 pacientes que se encontravam em grau I, II e III como "bom grau", tendo encontrado $13,8 \% \mathrm{em}$ grau I, 45,6\% em grau II e 40,6\% em grau III. Suriano ${ }^{101}$ mostrou, na sua tese de mestrado, que de um total de 322 pacientes operados no Hospital São Paulo da Escola Paulista de Medicina, 27,3\% encontravam-se em grau I, 42,6\% em grau II, 27\% em grau III e 2,5\% em grau IV no momento da internação. Sano \& Saito ${ }^{91}$ e Inagawa ${ }^{44}$ mostraram que a proporção dos pacientes internados em seus serviços, em graus I e II, variam de $54 \%$ a $80 \%$. Gallo, Oppitz e Santos ${ }^{32}$ encontraram 67\% de pacientes em grau I, $17 \%$ em grau II, $11 \%$ em grau III e 3\% em grau IV, no total de 107 pacientes.

A grande quantidade de escalas e suas variáveis trazem alguma dificuldade para a comparação de resultados. Kassel e cols. ${ }^{53}$, em estudo cooperativo de 3.521 pacientes, utilizaram uma variação da Escala de Glasgow em um total de sete itens. Jagger, Torner e $\mathrm{Kasse}^{45}$ mostraram que, usando um oitavo item nessa escala utilizada por Kassel e cols. ${ }^{53}$, que seria o relacionado à abertura ocular e a uma modificação na resposta motora da escala de Glasgow, haveria condições de se prognosticar, com razoável sucesso, cerca de $90 \%$ dos bons resultados e óbitos.

$\mathrm{Na}$ tabela 2, em que relacionamos a presença ou não de sangue na TC, constatamos que $12 \%$ dos pacientes apresentavam hematoma não compressivo, $21 \%$ apresentavam inundação ventricular, e $60 \%$, sangue em uma ou mais cisternas, no espaço subaracnóideo. O restante, $34 \%$ das $\mathrm{TC}$, não mostrava qualquer sinal de sangue nesse espaço. Portanto, em $66 \%$ dos casos houve a presença de sangue revelada pela TC. É importante salientar que 21 pacientes apresentaram sangue em múltiplos compartimentos ao exame de TC.

No presente momento, sabemos que o melhor método para o diagnóstico de HSA é por meio da TC, e o método é tanto mais preciso, quanto mais cedo for realizado. Kassel e cols. ${ }^{53}$ mostraram que, no dia da HSA, o índice de precisão é de $96,6 \%$; no primeiro dia, após a HSA, é de $92,8 \%$; no segundo dia, $84,8 \%$; no terceiro dia, $77,8 \%$, e assim sucessivamente.

Além disso, a TC ainda nos dá importantes informações a respeito da localização e da quantidade de sangue no espaço subaracnóideo, que é o mais importante fator no prognóstico do vasoespasmo, da presença de sangue intraventricular ou intracerebral, e, em alguns casos, da localização do aneurisma ${ }^{66}$. Kassel e cols. ${ }^{53}$ relataram $17 \%$ de hematomas intracerebral e intraventricular em seus pacientes. Le Roux e cols. ${ }^{62}$ apresentaram que $93,3 \%$ dos seus casos de HSA foram diagnosticados por TC; $45,1 \%$ dos casos apresentavam hemorragia intraventricular e 16,9\%, hemorragia intracerebral. Suriano $^{101}$, revisando $216 \mathrm{TC}$, confirmou que $65,7 \%$ mostravam a presença de HSA; desses, em 14,8\% havia hematoma intracerebral e, em 13,9\%, hemorragia intraventricular. Não foi constatada a presença de sangue ou qualquer outra anormalidade em 17,6\% dos casos.

As alterações na TC são encontradas em $64 \%$ a $85 \%$ dos casos de HSA; hematoma intracerebral é encontrado em $17 \%$ a $29 \%$ dos casos e a hemorragia intraventricular, em $7 \%$ a $38 \%{ }^{3,106}$. Jensen e cols. ${ }^{47}$ realizaram TC em $90 \%$ de seus pacientes com HSA, tendo encontrado resultados positivos em $100 \%$ dos exames efetuados.

A ressonância nuclear magnética (RNM) é, pelo menos, tão sensível quanto a TC para detectar uma HSA, se as aquisições adequadas de imagem forem utilizadas. No entanto, os altos custos do procedimento e a publicação de alguns trabalhos mostrando pequena sensibilidade da RNM em casos de HSA aguda têm limitado seu uso e, portanto, um estudo comparativo. Mas a alta resolução da RNM e as melhorias nas técnicas de angiografia por RNM deverão tornar esse exame mais importante no futuro ${ }^{14,69}$.

$\mathrm{Na}$ tabela 3, relacionamos os sítios de localização dos 103 aneurismas estudados, referentes aos 100 pacientes da nossa série. A angiografia cerebral dos quatro vasos foi realizada por cateterismo femural, tão logo as condições clínicas o permitiram.

Os riscos da angiografia cerebral em pacientes com aneurismas rotos são mínimos. As reações alérgicas com complicações estão na ordem de 1:50.000 casos e a morte após a injeção de contraste é ainda mais incomum $^{20}$. Outra complicação, felizmente muito rara, é a ruptura do aneurisma durante a angiografia ${ }^{6,104}$. Portanto, não há razão para que os pacientes não sejam investigados e, se o exame resultar negativo, perante as provas inequívocas da presença de HSA por presença de aneurisma, o procedimento deve ser repetido em um prazo de 20 a 30 dias.

$\mathrm{Na}$ comparação entre as complicações no pósoperatório e os resultados obtidos (Tabela 4), de um total de 13 pacientes que sofreram algum tipo de complicação, 4 desenvolveram hidrocefalia e tiveram que ser submetidos à derivação ventriculoperitonial (DVP); as infecções de pele ou ferida operatória foram responsáveis por $23 \%$ das complicações, enquanto a meningite bacteriana, a embolia pulmonar, o derrame pleural, as convulsões e a infecção respiratória foram responsáveis por $38,4 \%$ dessas complicações. Mesmo assim, 8 destes pacientes $(61,5 \%)$ evoluíram com resultado excelente; 1 paciente $(7,7 \%)$ com bom e 3 pacientes $(23 \%)$ com mau resultado, correspondendo a 2 casos de hidrocefalia derivados e 1 paciente que desenvolveu crises convulsivas no pós-operatório, que 
foram controladas pelo uso de anticonvulsivantes. Portanto, 13\% dos pacientes submetidos à cirurgia, dessa série apresentaram algum tipo de complicação pós-operatória, e somente 1 dos casos $(7,7 \%)$ foi diagnosticado como vasoespasmo, evoluindo para o óbito.

A hidrocefalia, citada por alguns autores ${ }^{61,73,85}$ pode acometer até $21 \%$ dos casos, chegando, na nossa série, a $4 \%$. No entanto, deve-se ressaltar que a maior incidência dessa complicação encontra-se nos pacientes em graus IV e V de Hunt \& Hess, enquanto nos graus I, II e III estaria situada abaixo dos $4 \%$.

A freqüência de vasoespasmo, presente em apenas um paciente na nossa série $(1 \%)$ e que evolui para o óbito, ficou abaixo dos resultados encontrados por $\mathrm{Kassel}^{53}$, que relata incidência em torno de 7,2\% e 13,5\%, provavelmente relacionada com o maior número de cirurgias realizadas com mais de 12 dias no nosso estudo.

A embolia pulmonar é uma das mais comuns alterações pulmonares associadas à HSA, junto à pneumonia e ao edema pulmonar ${ }^{53,104}$. Le Roux e cols. ${ }^{62}$, em sua série, relataram $0,9 \%$ de embolia pulmonar e $14,7 \%$ de pneumonias. Sundt e cols. ${ }^{100}$ tiveram 14 pacientes com evidências inequívocas de embolia pulmonar entre 722 casos ( $1,93 \%)$, enquanto $0,5 \%$ dos pacientes de Rinne ${ }^{88}$ tiveram como fator principal de causa mortis essa doença. Yasargil ${ }^{106}$ teve essa complicação em 3,2\% de seus pacientes, e Kassel e cols. ${ }^{53}$, em $0,8 \%$. A detecção precoce dessa alteração respiratória e o uso preventivo da subeparinização parece-nos o procedimento mais adequado para o tratamento dessa complicação pulmonar.

A infecção da ferida operatória é pouco relatada e somente um paciente $(0,4 \%)$ na série de Le Roux e cols. ${ }^{62}$ apresentou essa complicação, enquanto na nossa série o índice chegou a 3\%. Acreditamos ser devido ao fato de tratar-se de pacientes com baixo nível socioeconômico, más condições nutricionais e resistência imunológica inadequada.

A meningite bacteriana pós-operatória é citada por Yasargil ${ }^{106}$ em cerca de 3,8\% dos seus casos, enquanto Le Roux e cols. ${ }^{62}$ citam 2,2\%, mas todos com boa evolução, assim como na nossa série.

Já as crises convulsivas, observadas em um caso na nossa série (1\%), podem acometer de $4 \%$ a $27,5 \%$ dos pacientes ${ }^{55,77,84,94}$. Temos adotado como rotina o uso profilático de fenitoína e a manutenção dessa droga por seis meses, após os quais fazemos a retirada gradual. $\mathrm{O}$ uso de anticonvulsivantes para prevenir crises convulsivas por longo tempo (um ano ou mais) tem sido aceito na prática médica no pós-operatório. $\mathrm{O}$ uso rotineiro dessas medicações parece diminuir a freqüência das convulsões, mas não está bem definido que a terapia anticonvulsivante previna o desenvolvimento da epilepsia pós-operatória. Além disso, a administração de fenitoína, fenobarbital e outros anticonvulsivantes está associada a significativo risco de reações adversas, assim como reações colaterais $24,67,87,94$. Alguns autores já questionam este uso prolongado do anticonvulsivante e preconizam a retirada deste com sete dias de pós-operatório ${ }^{13}$.

O mecanismo do vasoespasmo e das lesões intrínsecas da parede arterial é desconhecido ${ }^{23}$. Essa afirmação é ainda bastante atual, não havendo uma definição sobre as causas e a melhor forma de tratamento dessa anormalidade vascular. A maior causa de mortalidade e morbidade em pacientes que sofreram HSA é o ressangramento e a isquemia cerebral tardia devido ao vasoespasmo. A presença de sangue no espaço subaracnóideo e coágulos nas cisternas basais são particularmente considerados como algumas das causas de vasoespasmo, identificado em $62 \%$ das angiografias realizadas entre o $4^{\circ}$ e o $12^{\circ}$ dia após a $\mathrm{HSA}^{66}$.

$\mathrm{Na}$ tabela 5, correlacionamos o vasoespasmo angiográfico com os resultados obtidos. Notamos que 23 pacientes $(23 \%)$ apresentaram vasoespasmo angiográfico. Destes, em 12 pacientes $(52,2 \%)$ o resultado foi excelente, em $5(21,7 \%)$ foi bom e, noutros 6 (26,1\%), mau.

A quantificação e a comparação do vasoespasmo angiográfico é extremamente controverso e são inúmeros os métodos empregados para a determinação desse fator prognóstico $33,56,90,91,100,108$, não havendo consenso, sendo um campo em aberto para novos trabalhos.

No nosso estudo, preferimos simplificar e adotar o critério da presença ou da ausência de vasoespasmo angiográfico, independentemente do local, da quantidade de vasos comprometidos e da intensidade da estenose arterial.

Auer e cols. ${ }^{10}$, em estudo prospectivo de 100 pacientes tratados cirurgicamente nas primeiras 72 horas, mostraram que em 76\% não havia nenhum tipo de vasoespasmo angiográfico, 15\% tinham menos de 50\% de estenose e $1 \%$ apresentava vasoespasmo difuso e de grande intensidade. Gurusinghe e Richardson ${ }^{36}$, em análise de 131 pacientes, relataram 51,9\% sem vasoespasmo, 31,3\% com o diâmetro do vaso reduzido a 50\%, $11,4 \%$ com redução de $50 \%$ a $75 \%$ e $5,4 \%$ com redução de mais de $75 \%$. Kikuchi e Watanabe ${ }^{56}$ relataram 40,9\% de vasoespasmo nos seus pacientes, e em 55,5\% destes os resultados foram excelentes, em $22,2 \%$ foram bons, em 11,2\% maus e 11,2\% evoluíram para o óbito. Georg e cols. ${ }^{33}$ apresentam resultados semelhantes.

Portanto, existe uma clara evidência da importância do vasoespasmo angiográfico nos resultados obtidos na cirurgia dos aneurismas intracranianos, mostrando que entre $10 \%$ e $15 \%$ dos pacientes com alterações angiográficas evoluem mal.

A tabela 6 refere-se ao número de dias transcorridos após a HSA, quando os pacientes foram submetidos à 
cirurgia e os resultados obtidos. Notamos que nenhum paciente da nossa série foi operado precocemente $(0 \mathrm{a}$ 3 dias), em função da própria sistemática do nosso serviço, que opta por esperar o melhor momento, isto é, quando as condições clínicas estão estabilizadas e o nível de consciência é satisfatório, independentemente dos dias transcorridos ${ }^{31}$. Entre o $4^{\circ}$ e o $7^{\circ}$ dia, foram operados 3 pacientes e todos evoluíram com excelente resultado. Daqueles operados entre o $8^{\circ}$ e $11^{\circ}$ dia, $50 \%$ evoluíram para resultado excelente e $50 \%$ para resultado bom. Nesta casuística, $91 \%$ dos pacientes foram operados a partir do $12^{\circ}$ dia e, nestes, a soma dos resultados bons e excelentes ficou entre $88 \%$ e $95 \%$; nesse grupo, ocorreu apenas um óbito, em doente operado no $18^{\circ}$ dia. No resultado global obteve-se excelente em $72 \%$, bom em $19 \%$ e mau em $9 \%$.

Diversos trabalhos têm procurado demonstrar a superioridade do tratamento precoce sobre o tratamento tardio dos aneurismas e vice-versa ${ }^{15,21,25,27,46,57,60,75,83,99}$. Os defensores da cirurgia precoce acreditam que ela proporcione diminuição nos fatores que determinam mortalidade e morbidade, como o ressangramento e a ocorrência de vasoespasmo ${ }^{50-54,64,65,75}$. Entretanto, não é aceita, de uma forma geral, a opinião quanto ao espectro de indicações para a cirurgia precoce, como as condições de admissão, o sítio do aneurisma e os fatores de riscos adicionais $1,11,17,26,28,38,51,53,54,68,95,98$. Autores como Sundt e cols. ${ }^{100}$ afirmam que o momento da intervenção cirúrgica é, em geral, determinado pela condição neurológica do paciente e pela intensidade dos sinais meníngeos, incluindo febre, e postergam as cirurgias para 7 a 9 dias após a HSA. Em 848 doentes com aneurismas operados em 24 hospitais universitários da Alemanha, $50,7 \%$ foram operados 15 dias após a HSA e $38,6 \%$ entre o $5^{\circ}$ e o $15^{\circ}$ dia, enquanto somente $11 \%$ sofreram intervenção cirúrgica entre 0 e 3 dias $^{47}$. Kassel e cols. ${ }^{54}$ concluíram que a cirurgia tardia (mais de 10 dias após a HSA) apresenta os melhores resultados, apesar do risco de $30 \%$ de mortalidade e morbidade durante a espera. Jomin e cols. ${ }^{48}$ apresentaram um índice de mortalidade de $60,6 \%$ nas cirurgias realizadas nas primeiras 48 horas, índice que cai para $10 \%$ após a primeira semana.

$\mathrm{Na}$ tabela 7, analisamos a influência da faixa etária nos resultados e notamos que 91 pacientes (91\%) de todas as idades obtiveram resultado excelente ou bom, e 9 pacientes $(9 \%)$ evoluíram com resultado mau. Setenta e um pacientes (71\%) estão situados entre os 30 e os 59 anos de idade e obtiveram resultado excelente ou bom. Os pacientes que evoluíram mal estão distribuídos em praticamente todas as faixas de idade e o único óbito ocorreu em paciente situado entre os $40 \mathrm{e}$ os 49 anos (paciente em grau III). É interessante notar que a soma dos percentuais de resultados bom e excelente varia de $87,5 \%$ a $100 \%$ em todas as faixas etárias, inclusive na oitava década, em que presumivelmente os resultados teriam que ser piores, em função das alterações vasculares encontradas nessa faixa etária. Se analisarmos os trabalhos publicados entre 1979 e $1984^{48,109}$, veremos que a mortalidade nas faixas etárias mais avançadas variava de $20 \%$ a $40 \%$. Mais recentemente, a idade tem sido considerada com menos preconceito, não constituindo fator discriminatório para a cirurgia, sendo vários os relatos de resultados satisfatórios ${ }^{29,35,43,93}$.

A correlação entre os resultados obtidos e o grau clínico (Tabela 8 ) dos pacientes, mostra que $46 \%$ dos pacientes estavam em grau I e se obteve $95,6 \%$ de resultado excelente e $4,4 \%$ de bom. Já nos pacientes em grau II (21\%) obteve-se 80,9\% de resultado excelente e $19,1 \%$ de bom; $29 \%$ dos pacientes encontravam-se em grau III, e somente $34,5 \%$ evoluíram com resultado excelente, 44,8\% com bom, e 20,7\% com resultado mau. Do total de 100 pacientes, somente 4 estavam em grau IV, obtendo-se $25 \%$ de resultado excelente e $75 \%$ de mau resultado.

Kikuchi e Watanabe ${ }^{56}$, em sua série, relataram que nos pacientes em graus I e II os resultados excelente e bom estão próximos aos $100 \%$, enquanto a morbidade e a mortalidade crescem nos graus III e IV. Sundt e cols. ${ }^{100}$ demonstraram que os melhores resultados são obtidos nos graus I e II. Auer e cols..$^{9}$ obtiveram 93\% de bons resultados nos graus I e II; Le Roux ${ }^{62}, 96,8 \%$ de bons resultados nos pacientes em grau I, 88,3\% em grau II e $81,3 \%$ em grau III.

Esses resultados corroboram uma tendência na literatura mundial que o grau clínico obtido pela escala de Hunt \& Hess é um dos fatores prognósticos mais importantes e mais fidedignos nos resultados no tratamento dos aneurismas intracranianos.

Quanto à influência da localização dos aneurismas nos resultados obtidos (Tabela 9), observamos que os percentuais de resultados excelente e bom se situam entre $90 \%$ e $100 \%$, em todos os sítios, com exceção dos aneurismas da artéria cerebral média nos quais esse valor se situa em 77,3\%. O mau resultado predominou entre os doentes com aneurismas da artéria cerebral média. O único óbito ocorreu em paciente com aneurisma da artéria comunicante anterior, o que representou $3,5 \%$ do total de pacientes com aneurisma dessa localização.

Jomin e cols. ${ }^{48}$ obtiveram resultados bom e excelente, variando de $64,6 \%$ a $100 \%$, e uma taxa de mortalidade entre $10 \%$ e $18 \%$, sendo a mortalidade dos pacientes com aneurismas da artéria cerebral média de 13,2\%. Sundt e cols. ${ }^{100}$ apresentaram percentagens de resultados bom e excelente, variando de $84 \%$ a 91\%, e também tiveram a maior taxa de morbidade e mortalidade nos aneurismas da artéria cerebral média (14\%). Em 1984, Jensen e cols.$^{47}$ mostraram resultados bom e excelente 
em $80 \%$ a $100 \%$ dos casos, mas para esses autores a mortalidade foi maior nos aneurismas da artéria carótida interna, que chegou a 25\%, assim como a de Yoshimoto e cols. ${ }^{109}$, com $6,9 \%$.

Na tabela 10, após a conversão do grau clínico segundo nosso critério, a pontuação obtida pelos pacientes está correlacionada aos resultados obtidos, e notamos que $95,7 \%$ dos que obtiveram 4 como pontuação máxima tiveram resultado excelente e somente $4,3 \%$ tiveram resultado bom. Entre os que alcançaram 3 como pontuação, $80 \%$ obtiveram resultado excelente e $20 \%$, bom. Com a pontuação 2, apenas 34,5\% evoluíram para resultado excelente; $44,8 \%$ evoluíram para bom; 20,7\%, para mau. No entanto, dos que alcançaram a pontuação mínima 1 , somente $25 \%$ obtiveram resultado excelente e $75 \%$, mau. Dos 72 pacientes que tiveram resultado excelente, $80 \%$ e $95,7 \%$ haviam recebido, respectivamente, pontuação 3 e 4 , enquanto $25 \%$ e $34,5 \%$ haviam recebido pontuação 1 e 2 , respectivamente. Ressalte-se que os maus resultados da nossa casuística ocorreram somente nos pacientes que haviam recebido pontuação 1 e 2 .

São numerosos os métodos existentes para graduar as melhoras neurológicas dos pacientes após a instalação da HSA por aneurisma, e é importante estimar o nível clínico de cada paciente, porque isso será um bom referencial entre os resultados pós-cirúrgicos e a condição clínica após a HSA. Entre as escalas que têm sido utilizadas para essa avaliação, consideramos três como as mais importantes: a de Botterell e cols. ${ }^{16}$, de Hunt \& Hess $^{40}$ e a da World Federation of Neurological Surgeons ${ }^{22}$. Evidentemente, todas sofreram variações, acréscimos e sugestões mas, em essência, permanecem inalteradas. Atualmente, existe uma tendência pela utilização da classificação de Hunt \& Hess na maioria dos serviços neurocirúrgicos ${ }^{10,36,62}$.

Dentro da nossa proposta de avaliar quantitativamente o grau clínico do paciente, adotamos a escala de Hunt \& Hess, pontuando com nota máxima de 4 os pacientes em grau I de Hunt \& Hess; com nota 3 os pacientes em grau II; com nota 2 os pacientes em grau III; com nota 1 os pacientes em grau IV; e com nota 0 os pacientes em grau V. Cumpre ressaltar que não tivemos, na nossa série, nenhum paciente em grau $\mathrm{V}$, porque não acreditamos que esse tipo de paciente seja beneficiado com a cirurgia. Por outro lado, é bastante conhecido o resultado obtido em pacientes em graus I, II e III, sendo esses os pacientes que mais se beneficiam do tratamento cirúrgico ${ }^{62,79,89}$.

A tabela 11 relaciona os resultados do tratamento cirúrgico com os achados tomográficos no momento da internação e convertidos para a pontuação por nós proposta. Os pacientes que tiveram, nessa avaliação, a melhor nota, isto é, 3, também obtiveram os melhores resultados: resultado excelente em $85,3 \%$ deles, bom em $11,8 \%$ e mau em $2,9 \%$. O resultado dos que alcançaram pontuação 2 foi excelente em 78,3\%, bom em 13,5\% e mau em $8,2 \%$. Já dentre os que alcançaram pontuação 1, o resultado foi excelente em $64,7 \%$, bom em $11,8 \%$, e mau em 23,5\%. Dentre os de pontuação 0 , alcançaram resultado excelente somente $25 \%$, em $66,6 \%$ o resultado foi considerado bom e em $8,4 \%$, mau. Também aqui, cabe ressaltar que $80,5 \%$ dos pacientes que evoluíram com resultado excelente receberam pontuação 3 e 2 , enquanto $19,5 \%$ obtiveram pontuação 0 e 1 .

A TC precoce, após a HSA, é um importante critério de prognóstico, quando usada junto às condições clínicas do paciente $^{10}$. Essa afirmativa nos mostra o realce ocupado pela TC entre os fatores prognósticos da HSA e da cirurgia dos aneurismas cerebrais. Em decorrência, inúmeros têm sido os métodos para avaliar as informações fornecidas por este exame na conduta terapêutica e sua evolução $0^{2,25,30,37,74,81}$. Diante dessa falta de consenso na adoção de um critério de avaliação da $\mathrm{TC}$, referente à quantidade de sangue, sua localização e seus reflexos posteriores, preferimos idealizar um protótipo simplificado, de fácil memorização e que nos auxiliasse no prognóstico da HSA. A presença de hematoma intracerebral, com sangue no espaço subaracnóideo e sangue intraventricular, receberia pontuação 0; a presença de sangue intraventricular e no espaço subaracnóideo, receberia pontuação 1; somente a presença de sangue difuso no espaço subaracnóideo, a pontuação seria 2; e a ausência de sangue na TC, pontuação 3 .

Por esta pontuação em ordem crescente, podemos verificar que, teoricamente, o cérebro tem condições mais favoráveis de resistir e recuperar-se das agressões devastadoras da HSA e do vasoespasmo subseqüente quanto menor for a quantidade de sangue visível na TC e quanto menor for o número de compartimentos atingidos.

A tabela 12, que correlaciona a pontuação dada à angiografia cerebral, obtida o mais precocemente possível, e os resultados cirúrgicos, nos mostra que foi obtido resultado excelente em 59 pacientes $(76,6 \%)$ dentre os 77 que não apresentavam vasoespasmo angiográfico, contra $13(56,5 \%)$ dos 23 que apresentavam vasoespasmo na angiografia, uma diferença bastante significativa. Já nos pacientes que tiveram bom resultado, $21,7 \%$ tinham vasoespasmo angiográfico prévio, contra $18,2 \%$ sem vasoespasmo angiográfico.

Os maus resultados foram significativamente maiores nos pacientes cuja angiografia prévia mostrava vasoespasmo $(21,8 \%$ contra $5,2 \%$ nos pacientes que não tinham vasoespasmo). O único óbito ocorreu em paciente cujo vasoespasmo arterial era evidente.

"Embora a presença de vasoespasmo angiográfico exerça influência desfavorável, em nossa opinião, a presença ou a ausência de espasmo é muito menos 
importante que o grau clínico, e nós não hesitamos em proceder à cirurgia em um paciente alerta e na presença de espasmo angiográfico". Com essa afirmação, Krayenbühl ${ }^{59}$ iniciou, há 24 anos, os debates sobre a real influência do vasoespasmo angiográfico nos resultados finais.

A presença ou a ausência de vasoespasmo angiográfico foi confirmada no nosso serviço por um neurorradiologista e pelo autor (PG), atribuindo-se pontuação 0 aos que mostravam sinais claros de vasoespasmo e pontuação 1 aos que mostravam os vasos com calibre normal. Ficou evidente nos resultados obtidos neste trabalho que, dos 77 pacientes operados sem vasoespasmo angiográfico, somente $5,2 \%$ obtiveram mau resultado, enquanto $94,8 \%$ obtiveram resultados excelente ou bom. Já o resultado obtido nos 23 pacientes que foram operados com a angiografia mostrando a redução no calibre dos vasos, foi mau em $21,8 \%$ e bom ou excelente em somente $78,2 \%$.

A tabela 13 mostra a correlação da pontuação geral com os resultados obtidos. O resultado do tratamento em todos os pacientes que obtiveram a pontuação máxima 8 foi excelente, evoluindo sem nenhuma anormalidade; $84 \%$ dos que obtiveram a pontuação 7 , $91,3 \%$ dos que obtiveram a pontuação $6,75 \%$ com pontuação $5,50 \%$ com pontuação 4 e $33,3 \%$ com pontuação 2 obtiveram também resultado excelente.

Os casos que resultaram em pequenos déficits, mas que não impediram de retornar às suas atividades normais (resultado bom), concentraram-se dentre os de pontuação 4,3 , e 2 , com $33,3 \%, 58,3 \%$ e $33,3 \%$, respectivamente.

Os maus resultados ficaram restritos àqueles com pontuação $2,3,4$ e 5, com predomínio dos de pontuação 2 e $3, \operatorname{com} 33,3 \%$ e $41,7 \%$, respectivamente.

\section{Conclusões}

1. O vasoespasmo angiográfico é um fator importante nos pacientes que tiveram mau resultado $(26,1 \%)$.

2. A faixa etária não constitui um fator de risco na cirurgia dos aneurismas intracranianos, desde que o paciente esteja em boas condições clínicas.

3. O grau clínico exerce papel importante para a obtenção de excelentes resultados e bons resultados, sendo os graus I, II e III os que melhor evoluem.

4. Os pacientes com aneurismas da artéria cerebral média são os que apresentam a maior incidência de pequenas seqüelas.
5. Todos os pacientes que obtiveram pontuação máxima 8 , do nosso critério, tiveram excelente resultado.

6. A pontuação máxima isolada em um só critério de avaliação não constitui, necessariamente, um bom prognóstico.

7. Quanto maior a soma dos três critérios de avaliação, melhor o prognóstico cirúrgico.

\section{Referências}

1. ADAMS HP: Early management of the patient with recent aneurysmal subarachnoid hemorrhage. Stroke 17:1068-70, 1986.

2. ADAMS HP, KASSEL NF, TORNER JC: Use fulness of computed tomography in predicting outcome after aneurysmal subarachnoid hemorrhage: a preliminary report of the Cooperative Aneurysm Study. Neurology 35:1263-7, 1985.

3. ADAMS HP, KASSEL NF, TORNER JC, SAHS AL: $C T$ and clinical correlations in recent subarachnoid hemorrhage: a preliminary report of the Cooperative Aneurysm Study. Neurology 33:981-8, 1983.

4. ADAMS CB, LOACH AB, O'LAOIRE SA: Intracranial aneurysms: analysis of results of microneurosurgery. Brit Med J 2:607-9, 1976

5. AMACHER AL, FERGUSON GG, DRAKE CG, GIRVIN JP, BARR HWK: How old people tolerate intracranial surgery for aneurysm. Neurosurgery 1:242-4, 1977.

6. AOYAGI N, HAIAKAWA I: Rerupture of intracranial aneurysms during angiography. Acta Neurochir (Wien) 98:141-7, 1989.

7. AUER LM: Acute operation and preventive nimodipine improve outcome in patients with ruptured cerebral aneurysms. Neurosurgery 15:57-66, 1984.

8. AUER LM: Preventive nimodipine and acute aneurysm surgery. Heading for the control of complications after aneurysmal subarachnoid hemorrhage. Neurochirurgia 28 (suppl)1:87-92, 1985.

9. AUER LM, BRANDT L, EBELING U, GILSBACH J, GROEGER U, HARDERS A, LJUNGGREN B, OPPEL F, REULEN HJ, SÄVELAND H: Nimodipine and early aneurysm operation in good condition SAH patients. Acta Neurochir (Wien) 82:7-13, 1986.

10. AUER LM, SCHNEIDER GH, AUER T: Computerized tomography and prognosis in early aneurysm surgery. J Neurosurg 65:217-21, 1986.

11. AUSMANN JL, DIAZ FG, MALIK GM, ANDREWS BT, MCCORMICK PW, BALAKRISHNAN G: Management of cerebral aneurysms: further facts and additional myths. Surg Neurol 32:21-35, 1989.

12. AUSMANN JL, DIAZ FG, MALIK GM, FIELDING AS, SON CS: Current management of cerebral aneurysms: is it based on facts or myths? Surg Neurol 24:625-35, 1985.

13. BAKER CJ, PRESTIGIACOMO CJ, SOLOMON RA: Short-term perioperative anticonvulsivant prophylaxis for the surgical treatment of low-risk patients with intracranial aneurysms. Neurosurgery 37:863-71, 1995.

14. BLATTER DD, PARKER DL, AHN SS, BAHR AL, ROBINSON RO, SCHWARTZ RB, JOLESZ FA, BOYER RS: Cerebral MR angiograph with multiple overlapping thin slab acquisition. Part II - Early clinical experience. Radiology 183:379-89, 1992. 
15. BOHM E, HUGOSSON R: Results of surgical treatment of 200 consecutive cerebral arterial aneurysms. Acta Neurol Scand 46:43-52, 1970.

16. BOTTERELL EH, LOUGHEED WM, SCOTT JW, WANDEWATER SL: Hypothermia and interruption of carotid, or carotid and vertebral circulation in the surgical management of intracranial aneurysms. J Neurosurg 13:1-42, 1956.

17. CHYATTE D, FODE NC, SUNDT TM: Early versus late intracranial aneurysm surgery in subarachnoid hemorrhage. J Neurosurg 69:326-31, 1988.

18. CROWELL RM, ZERVAS NT: Management of intracranial aneurysm. Med Clin North Am 63:695713, 1979

19. DERUTY R, MOTTOLESE C, PELISSOU-GUYOTAT I, SOUSTIEL JF: Management of the ruptured intracranial aneurysm - Early surgery, late surgery or modulated surgery? Acta Neurochir (Wien) 113:1-10, 1991.

20. DION JE, GATES PC, FOX AJ, BARNETT HJM, BLOM $\mathrm{RJ}$ : Clinical events following neuroangiography. A prospective study. Stroke 18:997-1004, 1987.

21. DISNEY L, WEIR B, PETRUK K: Effect on management mortality of a deliberate policy of early operation on supratentorial aneurysms. Neurosurgery 20:695-701, 1987.

22. DRAKE C: Report of World Federation of Neurological Surgeons Committee on a universal subarachnoid hemorrhage grading scale. J Neurosurg 68:985-6, 1988.

23. ECKER A, RIEMENSCHNEIDER PA: Artheriographic demonstration of spasm of the intracranial arterias with special reference to saccular arterial aneurysms. J Neurosurg 8:660-7, 1951.

24. ENGEL JR. J: Seizures and epilepsy. Philadelphia, FA Davis, 1989, pp 380-404.

25. FISCHER CM, ROBERSON GH, OJEMANN RG: Cerebral vasospasm with ruptured saccular aneurysms: the clinical manifestations. Neurosurgery 1:245-8, 1977.

26. FLAMM ES: The timing of aneurysm surgery. Clin Neurosurg 33:147-58, 1986.

27. FLEISCHER AS, TINDAL GT: Cerebral vasospasm following aneurysms rupture. A protocole for therapy and prophylaxis. J Neurosurg 52:149-52, 1980.

28. FRECKMANN N, NOLL M, WINKLER D, NOWAK G, REHN H, NEUSS M, HERMANN HD: Does the timing of aneurysm surgery neglect the real problems of subarachnoid haemorrhage? Acta Neurochir (Wien) 89:91-9, 1987

29. FRIDRIKSSON SM, HILLMAN J, SÄVELAND H, BRANDT L: Intracranial aneurysm surgery in the 8th and 9th decades of life: impact on population-based management outcome. Neurosurgery 37:627-32, 1995.

30. FUJITA S: Computed tomographic grading with Hounsfield number related to delayed vasospasm in cases of ruptured cerebral aneurysm. Neurosurgery 17:609-12, 1985.

31. GALLO P, BONATELLI AP, FERRAZ AP, BRAGA FM: Aneurismas da artéria cerebral média. Estudo comparativo entre duas vias de abordagem: via subfrontal e frontotemporoesfenoidal. Arq Bras Neurocir 12:77-104, 1993

32. GALLO P, OPPITZ PP, SANTOS AJ: Aneurismas intracranianos. Análise de 110 casos operados. Mom \& Perspec Saúde 4:49-52, 1990

33. GEORG B, ZERAH M, DEMATONS C, COPHIGNON J: L'anévrysme artériel intra-crânien. Une origine et un pronostic différents chez l'homme et chez la femme. Neurochirurgie 33:196-200, 1987.

34. GILSBACH JM: Nimodipine in the prevention of ischaemic deficits after aneurysmal subarachnoid haemorrhage. An analysis of recent clinical studies. Acta Neurochir (Wien) 45 (suppl):41-50, 1988.

35. GILSBACH JM, HARDERS AG, EGGERT HR, HORNYAK ME: Early aneurysm surgery: a 7 year clinical practice report. Acta Neurochir (Wien) 90:91 102, 1988

36. GURUSINGHE NT, RICHARDSON AE: The value of computerized tomography in aneurysmal subarachnoid hemorrhage. The concept of the CT score. J Neurosurg 60:763-70, 1984.

37. HIJDRA MD, BROUWERS PJAM, VERMEULEN M, VAN GIJN J: Grading the amount of blood on computed tomograms after subarachnoid hemorrhage. Stroke 21:1156- 61, 1990.

38. HILLMAN J, VON ESSEN C, LESZNIEWSKI W: Results of treatment for cerebral saccular aneurysms in a small neurosurgical unit-evaluation of early operation and nimodipine treatment. Acta Neurochir (Wien) 94:28-31, 1988.

39. HUGOSSON R: Intracranial arterial aneurysms: considerations on the upper age limit for surgical treatment. Acta Neurochir (Wien) 28:157-64, 1973.

40. HUNT WE, HESS RM: Surgical risk as related to time of intervention in the repair of intracranial aneurysms. J Neurosurg 46:14-20, 1968.

41. INAGAWA T: Multiple intracranial aneurysms in elderly patients. Acta Neurochir (Wien) 43:163-9, 1978.

42. INAGAWA T: Management outcome in the elderly patient following subarachnoid hemorrhage. J Neurosurg 78:554-61, 1993.

43. INAGAWA T, KAMIYA K, OGASAWARA H: Management of elderly patients with aneurysmal subarachnoid hemorrhage. J Neurosurg 69:332-9, 1988.

44. INAGAWA T, KAMIYA K, OGASAWARA H, YANO T: Rebleeding of ruptured intracranial aneurysms in the acute stage. Surg Neurol 28:93-9, 1987.

45. JAGGER J, TORNER JC, KASSEL NF: Neurologic assessment of subarachnoid hemorrhage in a large patients series. Surg Neurol 32:327-33, 1989.

46. JANE JA, WINN HR, RICHARDSON AE: The natural history of intracranial aneurysms. Rebleeding rates during the acute and long-term period and implications for surgical management. Clin Neurosurg 24:176-84, 1977.

47. JENSEN HP, KRAUS E, SCHEIL F: Surgical results in 1045 cases of intracranial aneurysms. S Afr J Surg 22:3-10, 1984.

48. JOMIN M, LESOIN F, LOZES G: Prognosis with 500 ruptured and operated intracranial aneurysms. Surg Neurol 21:13-8, 1984

49. KASSEL NF: The natural history and treatment outcome of SAH: comments derived from the National Cooperative Aneurysm Study Proceedings. Puerto Rico, 1983, pp 67.

50. KASSEL NF, DRAKE CG: Timing of aneurysm surgery. Neurosurgery 10:514-9, 1982.

51. KASSEL NF, TORNER JC: Aneurysmal rebleeding. A preliminary report from the Cooperative Aneurysm Study. Neurosurgery 13:479-81, 1983.

52. KASSEL NF, TORNER JC: The international cooperative study on timing of aneurysm surgery An update. Stroke 15:566-70, 1984.

53. KASSEL NF, TORNER JC, HALEY EC, JANE JA, ADAMS HP, KONGABLE GL: The International 
Cooperative Study on the timing of aneurysm surgery. Part 1: Overall management results. J Neurosurg 73:18-36, 1990 .

54. KASSEL NF, TORNER JC, JANE JA, HALEY EC, ADAMS HP: The International Cooperative Study on the timing of aneurysm surgery. Part 2: Surgical results. J Neurosurg 73:37-47, 1990.

55. KERANEN T, TAPANINAHO A, HERNESNIEMI J, VAPALAHTI M: Late epilepsy after aneurysms operations. Neurosurgery 17:897-900, 1985.

56. KIKUCHI K, WATANABE K: Modified bifrontal interhemisferic approach to aneurysms of the anterior communicating artery with the use of a trephine craniotomy. A review of personal experience with 25 cases. Acta Neurochir (Wien) 125:127-31, 1993.

57. KOOS WT, PERNECZKY A: Timing of surgery for ruptured aneurysms experience from 800 consecutives cases. Acta Neurochir (Wien) 63:12533, 1982.

58. KOSNIK EJ, HUNT WE: Postoperative hypertension in the management of patients with intracranial arterial aneurysms. J Neurosurg 45:148-54, 1976

59. KRAYENBÜHL HA, YASARGIL MG, FLAMM ES, TEW $\mathrm{JH}$ : Microsurgical treatment of intracranial saccular aneurysms. J Neurosurg 37:678-86, 1972.

60. KRUPP W, HEIENBROK W, MÜKE R: Management results attained by predominantly late surgery for intracranial aneurysms. Neurosurgery 34:227-34, 1994.

61. KUSSKE JA, TURNER PT, OJEMANN GA, GARRIS $A B$ : Ventriculostomy for the treatment of acute hydrocephalus following subarachnoid hemorrhage. J Neurosurg 38:591-5, 1973.

62. LE ROUX P, ELLIOTT JP, DOWNEY L, NEWELL DW, GRADY MS, MAYBERG MR, ESKRIDGE JM, WINN HR: Improved outcome after rupture of anterior circulation aneurysms: a retrospective 10 -year review of 224 good-grade patients. J Neurosurg 83:394-402, 1995.

63. LJUNGGREN B, SÄVELAND H, BRANDT L, USKI T: Aneurysmal subarachnoid hemorrhage. Surg Neurol 22:435-8, 1984

64. LJUNGGREN B, SÄVELAND H, BRANDT L, ZYGMUNT $S$ : Early operation and overall outcome in aneurysmal subarachnoid hemorrhage. J Neurosurg 62:547-51, 1985.

65. LOCKSLEY HB: Natural history of subarachnoid hemorrhage, intracranial aneurysms and arteriovenous malformations. J Neurosurg 25:321-68, 1966.

66. MACDONALD RL, WEIR B: Perioperative care following aneurysmal subarachnoid hemorrhage. In Schmidek $\mathrm{HH}$, Sweet WH (ed): Operative Neurosurgical Techniques. New York, Saunders, 1995, pp 937-55.

67. MANON-ESPAILLAT R, BURNSTINE TH, REMLER B, REED RC, OSORIO I: Antiepileptic drug intoxication: factors and significance. Epilepsia 32:96-100, 1991.

68. MARSH H, MAURICE-WILLIAMS RS, LINDSAY KW: Differences in the management of ruptured intracranial aneurysms: a survey of practice amongst British Neurosurgeons. J Neurol Neurosurg Psychiatry 50:965-70, 1987.

69. MATSUMARA K, MATSUDA M, HANDA J, TODO G: Magnetic resonance imaging with aneurysmal subarachnoid hemorrhage. Comparison with computed tomography scan. Surg Neurol 34:71-8, 1990.

70. MAURICE-WILLIAMS RS, MARSH H: Ruptured intracranial aneurysms: the overall effect of treatment and the influence of patient selection and data presentation on the reported outcome. J Neurol Neurosurg Psychiatry 48:1208-12, 1985.

71. MCKISSOC W, PAYNE KWE, WALSH LS: An analysis of the result of treatment of ruptured intracranial aneurysms. J Neurosurg 13:762-76, 1960.

72. MCKISSOCK W, RICHARDSON A, WALSH LS: Middle cerebral artery aneurysms: further results in the controled trial of conservative and surgical treatment of ruptured intracranial aneurysms. Lancet 2:417-21, 1962

73. MILHORAT TH: Acute hydrocephalus after aneurysmal subarachnoid hemorrhage. Neurosurgery 20:15-20, 1987.

74. MOHSEN F, POMONIS S, ILLINGWORTH R: Prediction of delayed cerebral ischaemia after subarachnoid haemorrhage by computed tomography. J Neurol Neurosurg Psychiatry 47:1197-202, 1984.

75. MULLAN S, HANLON K, BROWN F: Management of 136 consecutive supratentorial berry aneurysms. J Neurosurg 49:794-804, 1978.

76. NAKAGAWA T, HASHI K: The incidence and treatment of asymptomatic unruptured cerebral aneurysms. J Neurosurg 80:217-23, 1994.

77. NORTH JB, PENHAL RK, HANIEH A, FREWIN DB, TAYLOR WB: Phenytoin and postoperative epilepsy: a double blind study. J Neurosurg 58:672-7, 1983.

78. ÖHMAN J, HEISKANEN O: Effect of nimodipine on the outcome of patients after aneurysmal subarachnoid hemorrhage and surgery. J Neurosurg 69:683-6, 1988.

79. ÖHMAN J, SERVO A, HEISKANEN O: Risk factors for cerebral infiltration in good-grade patients after aneurysmal subarachnoid hemorrhage and surgery: a prospective study. J Neurosurg 74:14-20, 1991.

80. O'SULLIVAN MG, DORWARD N, WHITTLE IR, STEERS AJW, MILLER JD: Management and longterm outcome following subarachnoid hemorrhage and intracranial aneurysm surgery in elderly patients: an audit of 199 consecutive cases. Brit J Neurosurg 8:23-30, 1994.

81. PASQUALIN A, ROSTA L, DA PIAN R, CAVAZZANI P, SCIENZA R: Role of computed tomography in the management of vasospasm after subarachnoid hemorrhage. Neurosurgery 15:344-53, 1984.

82. PHILLIPS LH, WHISNANT JP, O'FALLON WM: The unchanging pattern of subarachnoid hemorrhage in a comunity. Neurology 30:1034-40, 1980.

83. POST KD, FLAMM ES, GOODGOLD A: Ruptured intracranial aneurysms. Case morbidity and mortality. J Neurosurg 46:290-5, 1977.

84. RABINOWICZ AL, GINSBERG DL, DEGIORGIO CM, GOTT PS, GIANOTTA SL: Unruptured intracranial aneurysms: seizures and antiepileptic drug treatment following surgery. J Neurosurg 75:371-3, 1991.

85. RAIMONDI AJ, TORRES H: Acute hydrocephalus as a complication of a subarachnoid hemorrhage. Surg Neurol 1:23-6, 1973

86. RASMUSSEM PH, BUSCH H, HAASE J, HAUSEN J, HARNNSEN A, KNUDSEN V, MARCUSEN E, OLSEN RB, ROSENARN J, SCHMIDT K, VALDBY B, HAUSEN L: Intracranial saccular aneurysms. Results of treatment in 851 patients. Acta Neurochir (Wien) 53:117, 1980.

87. RIMMER EM, RICHENS A: Clinical pharmacology and medical treatment. In Laidlow J, Richens A, Oxley J (ed): A Textbook of Epilepsy. New York, Churchil Livingstone, 1988, pp 421-83.

88. RINNE J, HERNESNIEMI J, NISKANEN M, VAPALAHTI M: Analysis of 561 patients with 690 
middle cerebral artery aneurysms: anatomic and clinical features as correlated to management outcome. Neurosurgery 38:2-11, 1996.

89. ROPPER AH, ZERVAS NT: Outocome 1 year after SAH from cerebral aneurysm: management morbidity, mortality and functional status in 112 consecutive good-risk patients. J Neurosurg 60:909-15, 1984.

90. SANCHEZ R, SPELLMAN JP: Radiologic features of cerebral vasospasm. Neurosurg Clin N Am 5:289-306, 1990.

91. SANO K, SAITO I: Timing and indication of surgery for ruptured intracranial aneurysms with regard to cerebral vasospasm. Acta Neurochir (Wien) 41:4960, 1978.

92. SARNER M, ROSE FC: Clinical presentation of ruptured intracranial aneurysm. J Neurol Neurosurg Psychiatry 30:67-70, 1967.

93. SÄVELAND H, HILLMAN J, BRANDT L, EDNER G, JAKOBSSON KE, ALGERS G: Overall outocome in aneurysmal subarachnoid hemorrhage. A prospective study from neurosurgical units in Sweden during a 1year period. J Neurosurg 76:729-34, 1992.

94. SBEIH I, TAMAS LB, O'LAOIRE SA: Epilepsy after operation for aneurysms. Neurosurgery 19:784-8, 1986.

95. SEILER RW, REULEN HJ, HUBER P, GROLIMUND P, EBELING U, STEIGER HJ: Outcome of aneurysmal subarachnoid hemorrhage in a hospital population: a prospective study including early operation, intravenous nimodipine, and transcranial Doppler ultrasound. Neurosurgery 23:598-604, 1988.

96. SERBINENKO FA: Six hundred endovascular neurosurgical procedures in vascular pathology. A ten-year experience. Acta Neurochir (Wien) 28:31021, 1979.

97. SMITH RR, MILLER JD: Pathophisiology and clinical evaluation of subarachnoid hemorrhage. In Youmans JR (ed): Neurological Surgery. 3 ed. Philadelphia, Saunders, 1990, pp 1644-60.

98. SOLOMON RA, FINK ME, LENNIHAN L: Early aneurysm surgery and prophylactic hypervolemic hypertensive therapy for the treatment of aneurysmal subarachnoid hemorrhage. Neurosurgery 23:699-704, 1988.

99. SUNDT JR. TM: Cerebral vasospasm following subarachnoid hemorrhage evolution, management and relationship to timing of surgery. Clin Neurosurg 24:228-39, 1977.
100. SUNDT JR. TM, KOBAYASHI S, FODE NC, WHISNANT JP: Results and complications of surgical management of 809 intracranial aneurysms in 722 cases. Related and unrelated to grade of patient, type of aneurysm, and timing of surgery. J Neurosurg 56:753-68, 1982

101. SURIANO IC: Aneurismas intracranianos. Estudo de 350 pacientes operados. Tese de Mestrado, Escola Paulista de Medicina, São Paulo, 1992.

102. TEASDALE G, JENNETT B: Assessment of coma and impaired consciousness. A practical scale. Lancet 81-3, 1974.

103. TORNER JC, KASSEL NF, WALLACE RB, ADAMS HP: Preoperative prognostic factors for rebleeding and survival in aneurysm patients receiving antifibrinolytic therapy: report of the Cooperative Aneurysm Study. Neurosurgery 9:506-13, 1981.

104. WEIR B: Aneurysms affecting the nervous system. Baltimore, Willians \& Wilkins, 1987, pp 671.

105. WINN HR, RICHARDSON AE, O'BRIEN W: Long-term prognosis in untreated cerebral aneurysms: late morbidity and mortality. Ann Neurol 4:418-6, 1978.

106. YASARGIL MG: Microneurosurgery. Stuttgart, Thieme, 1984, vol 1 e 2.

107. YASARGIL MG, GASSER JC, HODOSH RM, RANKIN TV: Carotid-ophtalmic aneurysms: direct microsurgical approach. Surg Neurol 8:155-65, 1977.

108. YOSHIMOTO Y, KWAK S: Age-related multifactorial causes of neurological deterioration after early surgery for aneurysmal subarachnoid hemorrhage. J Neurosurg 83:984-8, 1995.

109. YOSHIMOTO T, UCHIDA K, KANEKO U, KAYAMA U, SUZUKI J: An analysis of follow-up results of 1000 intracranial saccular aneurysms with definitive surgical treatment. J Neurosurg 50:152-7, 1979.

Original recebido em maio de 2000

Aceito para publicação em junho de 2000

\section{Endereço para correspondência:}

Pasquale Gallo

Rua Tobias da Silva 203, cj. 203

CEP 90570-020 - Porto Alegre, RS 